\title{
The observability analysis of distribution network with distributed generation
}

\author{
Xu Zhu ${ }^{1, a}$ \\ ${ }^{1}$ School of Electrical and Electronic Engineering, North China Electric Power University, Baoding \\ 071003, China; \\ a974887035@qq.com
}

Keywords: Distribution network, observability analysis, distributed generation, meter placement

\begin{abstract}
To meet the demand of distribution network state estimation containing various distributed generations (DGs), an observability analysis model is proposed. In the model, distribution network nodes are divided into two types, nodes connected with DGs and nodes not connected with DGs to calculate their power. Solving measurement equation, typical load patterns can be obtained, which determine the observability of a network; Meter placement is optimized as the graph partitioning algorithm is used in the weighted distribution network with DGs. Simulations with Modified IEEE 13-bus system and IEEE 33-bus system added DGs are carried out to illustrate the presented method is economical and feasible.
\end{abstract}

\section{Introduction}

In order to meet the requirements of environmental protection and resources conservation, smart distribution network has become a highlighted topic. DGs are expected to play a significant role in the prospective distribution system.

This paper presents the various models of DGs adapted to power calculation whose result needs substituting into the measurement equation. A universal observability analysis model based on typical load patterns is applied; optimization meter placement of distribution network with DGs model is established by the graph partitioning algorithm. IEEE bus systems connected with DGs can test and verify the models mentioned.

\section{The Establishment of the Text}

\subsection{Node power model.}

In order to calculate node power, distribution network nodes can be divided into two types, nodes attached to DGs and nodes not attached to DGs.

A. Nodes attached to DGs: Suppose the power factor $\cos \varphi$ is a constant.

load average active power $\quad \bar{P}=\frac{W}{24 D_{m}}$

load average reactive power $\quad \bar{Q}=\bar{P} \tan \varphi$

Above, $W$ is the power consumption of a certain period, $D_{m}$ is the number of days.

B. Nodes not attached to DGs: They are divided into 4 types, PQ type, P-Q(V) type, PI type and PV type according to the constant measurements of generation[4-5].

Following assumptions is made to simplify the problem:

(1) The phase difference of node voltage can be ignored.

(2) Regarding the non-PV type node voltage as that of the first node, $V_{0}$.

(3) Ignoring the next node power in the calculation of terminal node power.

In the following model, $P$ and $Q$ are active power and reactive power of node, $P_{G}$ and $Q_{G}$ are the active power and reactive power of produced by generator.

PQ-type DG model. Besides the previous PQ-type DG, permanent-magnet generators and 
inconstant speed wind power generator are considered as PQ type. The power of this type DGs only needs changing direction.

$$
\text { Node power: }\left\{\begin{array}{l}
P=-P_{\mathrm{G}} \\
Q=-Q_{\mathrm{G}}
\end{array}\right.
$$

P-Q(V)-type DG model. Constant speed wind power asynchronous generator is P-Q(V) type because the reactive power is a function of voltage.

$$
\text { Node power: }\left\{\begin{array}{l}
P=-P_{\mathrm{G}} \\
Q=-f(V)
\end{array}\right.
$$

PI-type DG model. Micro gas turbine, fuel cell and photovoltaic generation are usually regarded as PI type. The calculation of node power is the same as PQ type, and the reactive power can be obtained as follows:

$$
Q_{\mathrm{G}}=\sqrt{|I|^{2}\left(e^{2}+f^{2}\right)-P_{\mathrm{G}}^{2}}
$$

Where $I$ is constant node current, $e$ and $f$ are real and imaginary parts of node voltage.

PV-type DG model. Synchronous generation such as internal-combustion engine and traditional gas turbine is treated as PV type. The equations of node power is the same as PQ type. From the circuit theory, the distribution network with $S$ nodes can get $S-1=S$ independent loop. According to the loop-voltage equation, $Q_{\mathrm{G}}$ can be obtained[1].

In summary, the active power of DGs is easy to acquire, but reactive power needs calculating except PQ type. Besides, the calculation of PV type is particular because the other types as known quantity need to be obtained firstly.

\subsection{The observability analysis with DGs model.}

The power of two type nodes can be calculated, which provide known quantities for observability analysis. The users are classified into $N$ types by important degree grades. The number of measurement areas is $M$. Hypotheses are proposed below in modeling to simplify the equations:

A. The classification information of users is known.

B. Important degree grades are divided by active power.

C. Network Loss is ignored.

Measurement Equation[8]: $\quad \mathbf{Z}_{\mathrm{t}}=\mathbf{H X}_{\mathrm{t}}+\mathbf{V}_{\mathrm{t}}$

Above, $\mathbf{Z}_{\mathrm{t}}$ is the column vector of power measurements at time $t$ in $M$ areas, $\mathbf{H}$ is the active power matrix of $N$ types in $M$ areas, $\mathbf{X}_{t}$ the column vector of typical load patterns of $N$ types at time $t, \quad \mathbf{H X}_{\mathrm{t}}$ is the calculation of $\mathbf{Z}_{\mathrm{t}}, \mathbf{V}_{\mathrm{t}}$ is the vector of residuals.

There are three situations for Formula (2) as follows:

$\left\{\begin{array}{l}\text { observable, } \quad M>N \\ \text { observable, } M=N \& \text { Rank of Matrix H is N } \\ \text { unobservable, } \quad M<N\end{array}\right.$

Using the least square estimation, observable typical load patterns can be gotten.

The apparent power of node $j$ at time $t: \quad S_{j(t)}=\sum_{n=1}^{N} S_{j, n} \times x_{t(n)}$

Where $s_{j, n}$ is the apparent power of type $n$ in node $j$. The system can be observable.

According to the observability analysis with DGs, the minimal number of meters is $M=N$. A meter can be placed between the two nodes partitioned by graphpartitioning algorithm, which optimize the meter placement[8]. 


\section{Literature References}

The nodes where DG installed are classified into three types, PQ type, PV type, and P-Q(V) type[1-2]. By the simplified loop-voltage equations, the reactive power of PV-type DG can be attained[1]. However, PI-type DG are not proposed. The method of photovoltaic generation and micro gas turbine can calculate the reactive power of PI-type DGs[3-4].

Observability analysis is the prerequisite for state estimation which becomes the foundation and core of smart distribution grid. The previous research on observability analysis of distribution systems with DGs is only available for certain state estimation model[5-7]. The common solution of observability analysis is given when distribution network has no DG[8].

\section{The test on meter placement of distribution network with DGs}

Supposing node power are power of users, two tests of three models are carried out with the procedure.

Test 1: 11 nodes system with DGs is modified based on IEEE 13-bus system[5].

Test 2: IEEE 33-bus system added DGs.

The DG types and data are shown in table.1 below[4].

Table.1 Types and data of DGs

\begin{tabular}{ccc}
\hline Node attached to DG & Data of DG & Type of DG \\
\hline 800 & DFIG wind power generator: $\mathrm{P}=300 \mathrm{~kW}, \mathrm{Q}=100 \mathrm{kvar}$ & $\mathrm{PQ}$ \\
801 & Photovoltaic generation: $\mathrm{P}=150 \mathrm{~kW}, \mathrm{I}=15 \mathrm{~A}$ & $\mathrm{PI}$ \\
802 & Fuel cell generator: $\mathrm{P}=200 \mathrm{~kW}, \mathrm{~V}=0.98 \mathrm{kV}$ & $\mathrm{PV}$ \\
803 & Traditional wind power generator: $\mathrm{P}=300 \mathrm{~kW}, \mathrm{~s}=0.033$ & $\mathrm{P}-\mathrm{Q}(\mathrm{V})$ \\
\hline
\end{tabular}

Test 1. The assumed type information of users is shown in table. 2.

Table.2 Type information of users in Test 1

\begin{tabular}{cc}
\hline Type of users & Nodes \\
\hline 1 & $800,801,802$ \\
2 & $632,644,684$ \\
3 & $611,645,652,671$ \\
4 & $633,675,680$ \\
\hline
\end{tabular}

PQ-type, PI-type and PV-type DGs are set on node 633, node 632 and node 684, respectively. The weighted tree of 11 nodes distribution system with DGs are shown in Fig.1.

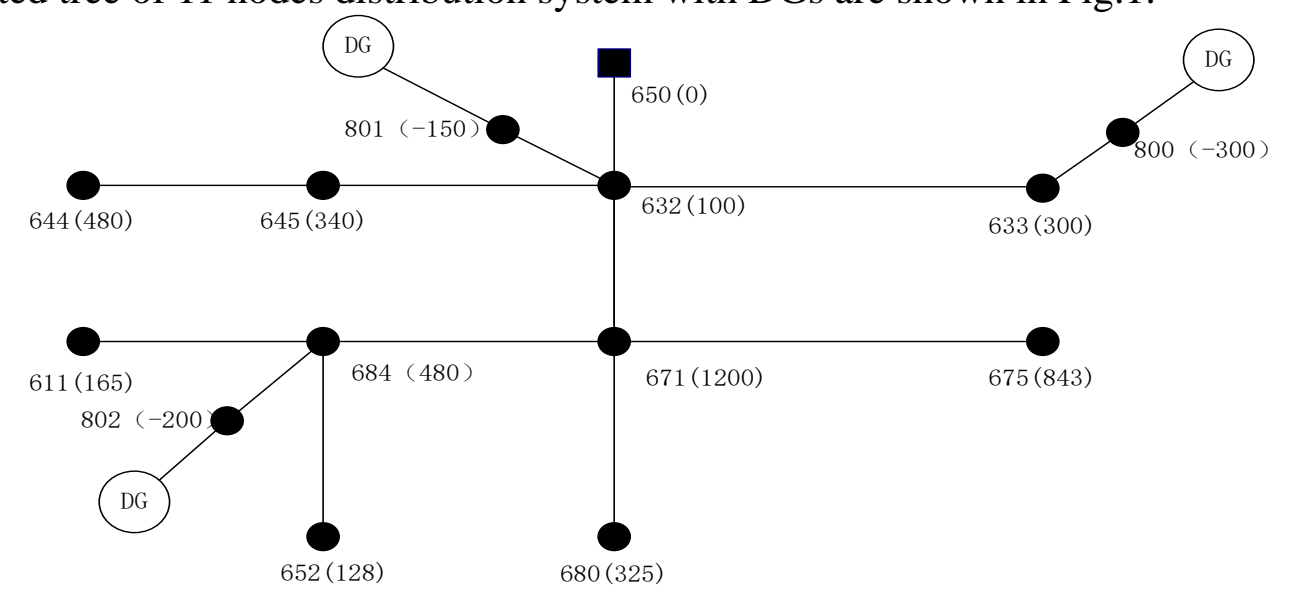

Figure. 1 The weighted tree of 11 nodes distribution system with DGs

The assumed type information of users is shown in table.2.

Table.2 Type information of users in Test 1

\begin{tabular}{cc}
\hline Type of users & Nodes \\
\hline 1 & $800,801,802$ \\
2 & $632,644,684$ \\
3 & $611,645,652,671$ \\
4 & $633,675,680$ \\
\hline
\end{tabular}


Analysis of test 1 results. Total weight of network shown in Fig.1 is 3713, and the number of measurement areas is 4 which is equal to the user-type number. Thus the weight of the areas should be close to $3713 / 4=928$. The Meter placement are shown in table.3.

Table.3 The results of meter placement in Test 1

\begin{tabular}{cccc}
\hline Measurement area & Meter position & Nodes of Measurement area & Weight \\
\hline 1 & $632-650$ & $632,633,650,671,680,800,801$ & 1475 \\
2 & $671-675$ & 675 & 843 \\
3 & $671-684$ & $611,652,684,802$ & 573 \\
4 & $632-645$ & 644,645 & 820 \\
\hline
\end{tabular}

Test 2. The weighted tree of IEEE 33-bus system added all the four types of DGs are shown in Fig.2, and the assumed type information of users is shown in table.4.

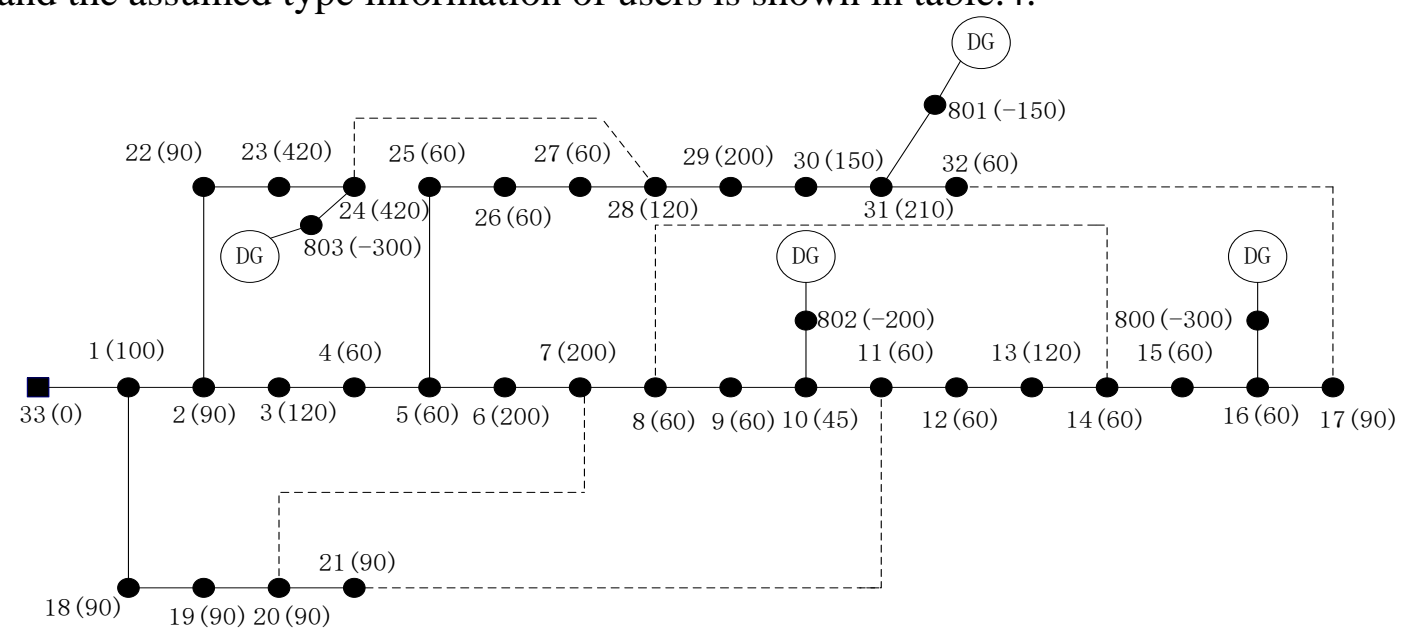

Figure.2 The weighted tree of IEEE 33-bus system added DGs

Table.4 Type information of users in Test 2

\begin{tabular}{cc}
\hline Type of users & Nodes \\
\hline 1 & $800,801,802$ \\
2 & $632,644,684$ \\
3 & $611,645,652,671$ \\
4 & $633,675,680$ \\
\hline
\end{tabular}

Analysis of test 2 results. The test 2 results are shown in table.5. Total weight of system shown in Fig. 2 is 2765, and the number of measurement areas is 6 . The weight of the areas should be close to $2765 / 6=461$, which is coincided with the results.

Table.5 The results of meter placement in Test 2

\begin{tabular}{cccc}
\hline Measurement area & Meter position & Nodes of Measurement area & Weight \\
\hline 1 & $31-32$ & $7,8,14,15,16,17,32,800$ & 290 \\
2 & $27-28$ & $28,29,30,31,801$ & 530 \\
3 & $4-5$ & $5,6,25,26,27$ & 440 \\
4 & $22-23$ & $23,24,803$ & 540 \\
5 & $1-18$ & $9,10,11,12,13,18,19,20,21,802$ & 505 \\
6 & $23-24$ & $1,2,3,4,22,23$ & 460 \\
\hline
\end{tabular}

\section{Summary}

This paper builds various DGs models to observability analysis. Under the preconditions of observability of distribution network, meter is placed using the graph partitioning algorithm. In the IEEE bus systems added DGs example, meter placement meets the balance need of users importance. The optimized meter placement makes the observability analysis more effective and economical, which is favorable for state estimation of distribution network with DGs. 


\section{References}

[1] H.N. Liu, T.J. Jing, H.C. Li, et al: Simplified Power Flow Calculation for Distribution Network With Distributed Generation Based on Loop Analysis, vol. 37 (2013) no. 4, p. 2-3.

[2] L.M. Zhang, W. Tang: Back/Forward Sweep Power Flow Calculation Method of Distribution Networks With DGs, vol.25 (2010) no. 8, p. 2.

[3] S.X. Wang, L.J. Huang, C.S. Wang, et al: Unbalanced Three Phase Power Flow Calculation of Distribution System, vol. 27 (2007) no. 8, p. 2.

[4] J. Dai, S. Wang, J.F. Zhu: Power Flow Method for Weakly Meshed Distribution Network With Distributed Generation, vol. 39 (2011) no. 10, p. 2-4.

[5] X.M. Tan: State Estimation of Distribution Systems With Distributed Generations (Master, Tianjin University, China 2008).

[6] S. Wang, Z.H. Jiang, J.F. Zhu, et al: State Estimation of Distribution Network Involving Distributed Generation, vol. 41 (2013) no. 13, p. 2.

[7] X.K. Cui: Study on Measurement Placement and State Estimation Algorithm in Distribution System With DGs (Master, Tianjin University, China 2012).

[8] D.Z. Chen, Z.Z. Guo: Distribution System Network Observability Analysis Based on Meter Placement, vol. 25 (2005) no. 12, p. 2-4. 